\title{
Comparison of three ultrasound-guided radial artery cannulation methods performed by anaesthesia residents: A prospective randomized controlled trial
}

\section{Yingli Cao}

Henan University People's Hospital https://orcid.org/0000-0002-6481-8691

Jingxin Su

Henan Provincial People's Hospital

Hang Fan

Henan Universiyi People's Hospital

Kang Kang

Henan University People's Hospital

Jiaqiang Zhang

Henan University People's Hospital

Mingzhu Cui ( $\nabla$ cuimingzhu78@163.com )

Henan University People's Hospital https://orcid.org/0000-0002-6711-6644

Research article

Keywords: Radial artery cannulation, Ultrasound, Residents

Posted Date: January 25th, 2021

DOl: https://doi.org/10.21203/rs.3.rs-152370/v1

License: (1) This work is licensed under a Creative Commons Attribution 4.0 International License.

Read Full License 


\section{Abstract}

\section{Background}

To compare the short-axis/out-of-plane (SAX), long-axis/in-plane (LAX) and oblique-axis/out-of-plane (OAX) approaches for radial artery catheterization performed by anaesthesia residents with limited experience.

\section{Methods}

Two hundred and sixteen adult patients were scheduled to undergo surgery requiring continuous arterial pressure monitoring. They were randomized to the SAX, LAX and OAX groups at a 1:1:1 ratio. Thirty-three anaesthesia residents performed the cannulation procedures. The operators received an explanation of the theoretical anatomy of the radial artery and the operating process of three approaches for ultrasoundguided cannulation of the radial artery and practised artery cannulation on a model of radial artery cannulation. The primary outcome was the success rate of cannulation, and the secondary outcomes included the first-attempt success rate in the model and patients, the imaging time, blood return time, cannulation time, total time and incidence of adverse events.

\section{Results}

The success rate of radial artery cannulation in the SAX group was significantly higher than that in the LAX group (71.4\% vs $49.2 \% ; P=0.009)$ and was clinically meaningfully higher than that in the OAX group (71.4\% vs 61.7\%; $P=0.229)$. The total time was shorter in the SAX group than in the LAX and OAX groups: 51 (59) s vs 113 (66) s vs 86 (61) s; $P=0.000$. The first-attempt success rate for the model did not differ among the three groups. In the patients, the first-attempt success rate in the SAX group was significantly higher than those in both the LAX and OAX groups ( $69.7 \%$ vs $24.2 \%$ vs $6.1 \% ; P=0.000$ ).

\section{Conclusion}

Anaesthesia residents can achieve a higher success rate, higher satisfaction rate and shorter procedure time by using the SAX approach than by using the LAX and OAX approaches for radial artery catheterization.

\section{Trial registrration}

Chinese Clinical Trial Registry,ChiCTR200030416. Registered 1 March 2020,http://www.chictr.org.cn/edit.aspx?pid=50193\&htm=4

\section{Background}

Invasive arterial pressure measurements can accurately and timely detect changes in blood pressure and are widely used in the perioperative period as a haemodynamic monitoring index. The radial artery is the 
most commonly used vessel for artery cannulation because of its anatomical accessibility, the dual arterial supply of the hand and the low complication rate[1]. Arterial cannulation allows beat-to-beat blood pressure measurement and blood sampling for blood gas analysis, helps medical personnel deal with emergencies quickly and reduces perioperative complications[2].

The traditional operation for radial artery cannulation is performed by palpating body parts to identify the pulse, which is mainly affected by the operator's sensation in his or her hand and experience. However, anatomical variations, obesity, hypotension, oedema, and atherosclerosis may render artery localization via palpation a difficult or even impossible task[3]. Recently, ultrasound-guided radial artery catheterization has offered clear benefits, such as an increased success rate, patient safety, and cost effectiveness, compared with traditional palpation. Preliminary training and familiarization with the ultrasound-guided technique is needed before applying it for radial artery catheterization, especially for inexperienced anaesthesiologists[4-5].

To our knowledge, there are three approaches for ultrasound-guided radial artery cannulation: the shortaxis/out-of-plane (SAX), the long-axis/in-plane (LAX) and the oblique-axis/out-of-plane (OAX) approaches, with different degrees of success for each approach[6.7]. These results corresponded to physicians with ultrasound experience, and the utility of these three techniques when used by nonexpert personnel has not been studied. Since a large fraction of cannulations are performed by non-experts, a better understanding of the value of these methods in novices is needed. Thus, we aimed to compare the success rate of radial artery cannulation in adult surgical patients performed by anaesthesia residents using the SAX, LAX or OAX approach.

\section{Methods}

The current prospective, randomized, observational study was approved by the Institutional Review Board of Henan Provincial People's Hospital in China (no. 2020-16; 20 January 2020) and was registered in the Chinese Clinical Trial Registry (ChiCTR200030416; 1 March 2020).

\section{Participants and randomization}

Patients of either sex, aged from 40-65 years, ASA status of I-III,with a BMI between $20-35 \mathrm{~kg} / \mathrm{m}^{-2}$ and who required continuous arterial pressure monitoring during their scheduled surgery were eligible to participate. Patients who had inflamed skin near the puncture site; patients in whom the skin colour did not return to normal within $10 \mathrm{~s}$ after Allen's test; patients who had peripheral arterial disease, abnormal coagulation function, recent arterial puncture $<1$ month earlier, hypertension and diabetes; and patients requiring emergency surgery were excluded from the study.

The patients were assigned by a randomized block design to three groups. We allocated patients at a 1:1:1 ratio with a computer-generated list of random numbers in blocks of three, with the results accessible to only research nurses. The sealed envelopes were opened immediately before the procedure. To prevent the anaesthesia residents' level of experience with the techniques from confounding the 
results, an allocation block was used to assign individual anaesthesia residents to each study procedure. Operators who participated in the study completed from one to three blocks.

The operators selected were anaesthesia residents with no more than one year of experience in blind palpation for radial artery cannulation and who previously performed ultrasound-guided radial artery cannulation fewer than five times in patients.

\section{Training phase}

Prior to the start of the study, we provided training to all participants, including a theoretical explanation of the anatomy of the radial artery and the operating processes for the three approaches for ultrasoundguided cannulation of the radial artery, as well as practice on a model of radial artery cannulation for approximately 2 hours. One teacher who was familiar with the three approaches demonstrated the three methods for everybody in steps, and the students practised under the guidance of the teacher to ensure that each resident was familiar with the operation steps. At the end of the training session, all participants had completed radial artery catheterization with the three different approaches, and the firstattempt success rate and satisfaction rate were recorded.

The radial artery puncture model was made of a rectangular, soft silicone material with excellent ultrasonic transmission qualities. The model had an electronic pump to keep the fluid flowing, when the needle penetrated the blood vessel, there was flashback of blood in the tail of the needle (Figure 1).

\section{Operation method}

Upon arrival to the operating room, the patients underwent oxygen saturation, electrocardiogram and noninvasive blood pressure monitoring, inhaled oxygen for $2 \mathrm{~L} / \mathrm{min}$.

\section{Positioning measurement}

In the supine position, the operator selected the side of the hand according to the surgical indication. The arm was abducted $90^{\circ}$, and a sterile pad was placed on the wrist to keep the back of the wrist at approximately $45^{\circ}[8]$. The probe was placed perpendicular to the radial artery, and the radial styloid process was the measuring point. Once the radial artery was identified, the cross-sectional view of the artery was frozen and saved to measure the diameter and depth of the artery. The same ultrasound device was used for the measurements (SONIMAGE HS1, KONICA MINOLTA, China), and the device had a linear transducer ( 5 to $13 \mathrm{MHz}$ and depth of $1 \mathrm{~cm}$ ). All the data were measured and recorded by the same person.

\section{Technique of radial artery cannulation}

After sterile skin preparation, radial artery cannulation was performed according to the grouping method in the sealed envelope. 
In the SAX group, the probe was placed perpendicular to the course of the artery, and the needle was directed perpendicular to the longitudinal axis of the probe. In the LAX group, the probe was placed parallel to the course of the artery, and the needle was directed parallel to the longitudinal axis of the probe. In the OAX group, the probe was positioned transversely perpendicular to the artery as in the SAX group and then rotated clockwise in situ by $60^{\circ}$ according to maximum visualization. After the arterial image was obtained, local anaesthesia was performed with $2 \%$ lidocaine. A $22-\mathrm{G}$ needle ( Sandy Ut 84070 , USA) was inserted at approximately $15-45^{\circ}$ into the skin until the tip was seen on the screen. After it penetrated the blood vessel and there was blood confluence in the tail of the needle, the position of the needle was adjusted and gently advanced slightly more distally under ultrasound guidance. Then, the ultrasound probe was placed down, the procedure was continued, and whether there was a waveform on the monitor was observed.

\section{Outcome evaluation}

The anaesthesia residents performed radial artery cannulation only once for each patient. Successful arterial cannulation was defined as the appearance of an arterial waveform after the catheter was connected to the transducer.The first attempt success rate was the rate of anaesthesia resident's first attempt on the patient and model using each apprach. The time needed for successful arterial cannulation in all patients was measured. The interval between the probe contacting the skin and the appearance of the arterial waveform was recorded as the total time of arterial cannulation. The interval between the probe contacting the skin and the disposable arterial needle contacting skin was recorded as the imaging time. The time from the needle contacting the skin to the first flashback of blood was recorded as the blood return time. The time from the first flashback of blood to the appearance of the arterial waveform on the monitor was recorded as the cannulation time. A total arterial cannulation time of more than 5 minutes and the presence of a non-arterial waveform were regarded as cannulation failure.

All the procedures were carried out under the guidance of an experienced anaesthesiologist. All failed procedures were completed by an expert anaesthesiologist. The primary outcome was the success rate of cannulation, and the secondary outcomes included the first-attempt success rate of cannulation in the model and patients (the success rate of the first operation for each approach), the imaging time, blood return time, cannulation time, total time and the incidence of adverse events. The adverse events included local haematoma and vasospasm. At the end of the study, the operator's level of satisfaction with the three methods was recorded. All data were recorded by the same anaesthesiologist who was not involved in the trial.

\section{Statistical analysis}

Sample size:

To calculate the sample size, 10 anaesthesia residents performed the procedure in 30 patients for a pilot trial. The patients were randomly divided into 10 blocks of three, and every block was assigned to 
individual anaesthesia residents using each technique. The success rate was $50 \%$ for $L A X, 80 \%$ for SAX, and $60 \%$ for OAX. We calculated that a total of 187 patients were needed for the three groups to compare these groups with $80 \%$ statistical power at a Bonferroni-adjusted $P$ value of 0.017 . Considering a $10 \%$ drop-out rate, the final sample size of this study was 207 , and 69 patients were needed for each group.

The data were recorded using IBM SPSS 23.0. Only patients in whom the needle could not be seen on ultrasound were excluded. The normally distributed continuous data are presented as the mean $\pm S D$, whereas the nonnormally distributed continuous data are presented as the median (interquartile range). Percentages were used to present the nominal and ordinal data, and the Kruskal-Wallis test was used for subsequent analysis. For the detection of significant differences among the three techniques, 1-way analysis of variance with Tukey's post hoc analysis or the Kruskal-Wallis $\mathrm{H}$ test was used as appropriate. Logistic regression analysis with a stepwise forward selection model was used to identify the independent covariates that were associated with the success rate. A $P$ value of less than 0.05 was considered significant.

\section{Results}

The patients were recruited between April 1, 2020 and July 28,2020. A total of 239 patients were included in the study, and the recruitment and group allocation results are illustrated in Fig. 2. Thirty-three anaesthesia residents participated in the training, and all of these residents enrolled in the study. They each performed 3 to 9 procedures (each method 1 to 3 times). The characteristics of the patients are summarized in Table 1. None of the patient characteristics significantly differed across groups.

Table 1

Baseline characteristics of patients

\begin{tabular}{|lllll|}
\hline & $\begin{array}{l}\text { Group SA } \\
(\mathbf{n}=\mathbf{7 0})\end{array}$ & $\begin{array}{l}\text { Group LA } \\
(\mathbf{n = 6 3 )}\end{array}$ & $\begin{array}{l}\text { Group OA } \\
(\mathbf{n}=68)\end{array}$ & $P$ \\
\hline Males(\%) & $32(46)$ & $34(54)$ & $33(49)$ & 0.579 \\
\hline Age(y) & $50 \pm 12$ & $52 \pm 10$ & $51 \pm 13$ & 0.510 \\
\hline BMI & $25 \pm 4$ & $25 \pm 4$ & $24 \pm 3$ & 0.995 \\
\hline HR(bpm) & $73 \pm 11$ & $74 \pm 12$ & $75 \pm 9$ & 0.525 \\
\hline SBP(mmHg) & $128 \pm 16$ & $131 \pm 17$ & $130 \pm 17$ & 0.504 \\
\hline DBP(mmHg) & $76 \pm 11$ & $76 \pm 10$ & $76 \pm 12$ & 0.995 \\
\hline Depth(mm) & $24 \pm 7$ & $25 \pm 7$ & $25 \pm 8$ & 0.769 \\
\hline Diameter (cm) & $21 \pm 4$ & $22 \pm 4$ & $20 \pm 3$ & 0.208 \\
\hline $\begin{array}{l}\text { Values are mean } \pm \text { SD or number(\%). BMl, body mass index; LAX, long-axis/in-plane;SAX,short- } \\
\text { axis/out-of-plane;OAX,oblique-axis/out-of-plane. }\end{array}$ & & \\
\hline
\end{tabular}


The first-attempt success rate for the model did not differ among the three groups $(36.4 \%$ vs $4.5 \%$ vs 9.1\%; $P=0.208$ ), when in the patients, the success rate in the SAX group was significantly higher than those in both the LAX and OAX groups ( $69.7 \%$ vs $24.2 \%$ vs $6.1 \% ; P=0.000)$. The satisfaction level of the anaesthesia residents was higher in the LAX group than in the SAX and OAX groups $(60.6 \%$ vs $30.3 \%$ vs 9.1\%; $P=0.000$ ) for the model, and the satisfaction level was higher in the SAX group than in the LAX and OAX groups $(60.6 \%$ vs $27.3 \%$ vs $12.1 \% ; P=0.000)$ in the patients, as presented in Table 2 .

Table 2

First attempt success and satisfication rate in model and patients

\begin{tabular}{|c|c|c|c|c|}
\hline & $\begin{array}{l}\text { Group SA } \\
(n=33)\end{array}$ & $\begin{array}{l}\text { Group LA } \\
(n=33)\end{array}$ & $\begin{array}{l}\text { Group OA } \\
(n=33)\end{array}$ & $P$ \\
\hline \multicolumn{5}{|l|}{ Satisfication } \\
\hline Mold,n(\%) & $10(30.3)^{b}$ & $20(60.6 \%)^{a}$ & $3(9.1 \%)^{\mathrm{ab}}$ & .000 \\
\hline Patient,n(\%) & $20(60.6 \%)^{b}$ & $9(27.3 \%)^{a}$ & $4(12.1 \%) \mathrm{a}$ & .000 \\
\hline \multicolumn{5}{|c|}{ First attempt success } \\
\hline Mold n (\%) & $10(36.4 \%)$ & $15(54.5)$ & $8(9.1 \%)$ & 0.208 \\
\hline Patient n (\%) & $23(69.7 \%)^{b}$ & $8(24.2 \%)^{a}$ & $2(6.1 \%)^{a b}$ & 0.000 \\
\hline \multicolumn{5}{|c|}{$\begin{array}{l}\text { Values are median (interquartile range) or number (\%).LAX, long-axis/in-plane;SAX,short-axis/out-of } \\
\text { plane;OAX,oblique-axis/out-of-plane. }\end{array}$} \\
\hline \multicolumn{5}{|c|}{ a indicates significant difference between group SAX and other group. } \\
\hline
\end{tabular}

Table 3 summarizes the results. The success rate of radial artery cannulation in the SAX group was significantly higher than that in the LAX group $(71.4 \%$ vs $49.2 \% ; P=0.009)$ and was clinically meaningfully higher than that in the OAX group $(71.4 \%$ vs $61.7 \% ; P=0.229)$. The imaging time was significantly longer in the LAX group than in both the SAX and OAX groups: 30 (17) s vs 10 (15) s vs 20 (14) $\mathrm{s} ; P=0.000$. The blood return time was significantly shorter in the SAX group than in both the LAX and OAX groups: 20 (22) s vs 51 (82) s vs 39 (46) s; $P=0.000$. The cannulation time did not statically significantly differ across groups: 20 (19) s vs 10 (13) s vs 14 (31) s; $P=0.066$. The total time was shorter in the SAX group than in both the LAX and OAX groups: 51 (59) s vs 113 (66) s vs 86 (61) s; $P=0.000$. The incidence of adverse events (haematoma, ischaemia) did not differ significantly among the three groups. 
Table 3

Procedural data

\begin{tabular}{|lllll|}
\hline & $\begin{array}{l}\text { Group SAX } \\
(\mathbf{n = 7 0 )}\end{array}$ & $\begin{array}{l}\text { Group LAX } \\
(\mathbf{n = 6 3 )}\end{array}$ & $\begin{array}{l}\text { Group OAX } \\
(\mathbf{n = 6 8 )}\end{array}$ & $P$ \\
\hline Success rate,n (\%) & $50(71.4 \%)^{\mathrm{b}}$ & $31(49.2 \%)^{\mathrm{a}}$ & $42(61.7 \%)$ & 0.03 \\
\hline Imaging time(s) & $10(11)^{\mathrm{b}}$ & $30(17)^{\mathrm{a}}$ & $21(15)^{\mathrm{ab}}$ & 0.000 \\
\hline Blood return time(s) & $20(22)^{\mathrm{b}}$ & $51(82)^{\mathrm{a}}$ & $39(46)^{\mathrm{a}}$ & 0.000 \\
\hline Cannulation time(s) & $20(19)$ & $10(13)$ & $14(31)$ & 0.066 \\
\hline Total time(s) & $51(59)^{\mathrm{b}}$ & $113(66)^{\mathrm{a}}$ & $86(61)^{\mathrm{a}}$ & 0.000 \\
\hline Adverse events & & & 0.653 \\
\hline Haematoma.n,(\%) & $6(8 \%)$ & $4(6 \%)$ & 0.154 \\
\hline Ischemia, $\mathrm{n}$ (\%) & $4(5 \%)$ & $2(3 \%)$ & \\
\hline $\begin{array}{l}\text { Values are median (interquartile range) or number (\%).LAX, long-axis/in-plane;SAX,short-axis/out-of- } \\
\text { plane;OAX,oblique-axis/out-of-plane. }\end{array}$ & & \\
\hline a indicates significant difference between group SAX and other group. & \\
\hline b indicates significant difference between group LAX and other group. & \\
\hline
\end{tabular}

Table 4 summarizes the association of factors with successful arterial cannulation. The cannulation approach was associated with the success rate. 
Table 4

Logistic Regression

\begin{tabular}{|lllll|}
\hline & OR & SE & $P$ vaule & $95 \% \mathrm{Cl}$ \\
\hline Males & 0.837 & 0.296 & 0.548 & $0.468-1.495$ \\
\hline Age $(\mathrm{y})$ & 0.985 & 0.013 & 0.236 & $0.960-1.010)$ \\
\hline BMI & 1.010 & 0.046 & 0.825 & $0.923-1.106$ \\
\hline HR(bpm) & 1.003 & 0.014 & 0.856 & $0.975-1.031$ \\
\hline SBP(mmHg) & 1.008 & 0.012 & 0.481 & $0.985-1.032$ \\
\hline DBP(mmHg) & 1.002 & 0.018 & 0.899 & $0.968-1.037$ \\
\hline Depth $(\mathrm{mm})$ & 0.149 & 1.886 & 0.313 & $0.004-6.005$ \\
\hline Diameter $(\mathrm{cm})$ & 4.887 & 3.885 & 0.683 & $0.002-9.892$ \\
\hline Method & & & 0.034 & \\
\hline SAX & 1 (ref.) & & & $1.261-5.281$ \\
\hline LAX & 2.581 & 0.365 & 0.009 & $0.832-3.342$ \\
\hline OAX & 1.667 & 0.355 & 0.149 & \\
\hline OR, odds ratio; SE, standard error; Cl, confidence interva; BMl, body mass index; ref, reference category; \\
LAX, long-axis/in-plane; SAX,short-axis/out-of-plane; OAX, oblique-axis/out-of-plane.
\end{tabular}

\section{Discussion}

In this study, we compared the application of three different ultrasound-guided radial artery cannulation approaches by anaesthesia residents without ultrasound experience. The SAX approach is considered to be an easier method than the LAX and OAX approaches because it is associated with a higher success rate, a shorter completion time and a higher satisfaction rate.

The practicability of the three approaches for radial artery cannulation in adults and children has been shown to be inconsistent across studies, and most studies have compared the LAX and SAX approaches under ultrasonic guidance. In a study by Berk et al[9]. the success rate of LAX was higher than that of SAX (76\% vs $51 \%, P<0.05)$, and the total cannulation time was shorter $(47 \pm 34 \mathrm{~s}$ vs. $24 \pm 17 \mathrm{~s}, P<0.05)$. Seith et al.[10] reported that there are no significant differences in the first-attempt success rate between the two approaches $(82.6 \%$ vs $80 \%, P=0.67)$. Abdalla et al.[6] described the OAX approach as a novel technique for radial artery cannulation and confirmed that it improved both the success rate and operator satisfaction level compared with other approaches in 2017. All participants in these studies were expert clinicians, and variations in clinician skill level can considerably affect the results. The diameter of the patient's artery, BMI, wrist positioning and other factors also affected the results[11.12]. To assess the 
pros and cons of the three approaches, we controlled for these influencing factors, as presented in Table 4. None of the operators had any experience in ultrasound-guided radial artery cannulation. A randomized block design was used, and each operator performed 3-9 operations to reduce intraindividual variation.

Blaivas et al[13] reported that the success rate for SAX was better than LAX for novices with the model, and most previous studies on ultrasound-guided vascular pathways focused on the SAX approach. A trial using a model verified that the visibility of the needle in the LAX approach is high, and both novice and veteran operators prefer the LAX approach, according to Stone et al[14]. The participants in our study were more inclined to use the LAX approach when practising with the model. The LAX approach affords improved visualization of the needle tip at the time of puncture, and the entire length of the needle can potentially be tracked on the ultrasound screen as it enters the blood vessel, so it is easier to use a cannula, and the operator is less likely to pierce the skin on the back or side walls[7]. However, when the participants in our study perform radial artery catheterization in patients, they preferred the SAX approach. The reason may be that the simulated blood vessels are easy to differentiate from other tissues, and the operator can see the relationship between the needle and blood vessels.

Ultrasound-guided radial artery cannulation is composed of three parts: image localization, puncture of the artery and cannula insertion into the blood vessel. In the SAX approach, the artery is easy to see, and the operator can easily align the cannula. For the LAX approach, the long axis of the whole artery needs to be visualized, and the surrounding muscle tissue and blood vessels are difficult to differentiate with ultrasound. It is difficult for operators to find complete arterial images, which leads to a significant increase in the imaging time of the LAX approach $(P=0.000)$. Second, the in-plane operation requires a higher level of hand-eye coordination by the operator, and it is difficult to algin the needle and artery in the same plane, so the blood return time was longer $(P=0.001)$.

The OAX approach is performed halfway between the short and long axes of the vessel, allowing visualization of the needle as it enters the vessel. Although the largest arterial image can be obtained by adjusting the probe, which is theoretically easier to enter the blood vessel, it requires additional dexterity and might not be easily adopted by novice residents[15]. Some studies have recommended that ultrasound guidance is used for central line placement to improve patient safety[16]. We placed the arterial image in the middle of the ultrasound screen, taking the centre line as the needle entry point; however, when the probe was rotated, the position of the artery in the image moved, and it was difficult for the operator to choose the needle point, which led to a longer blood return time for this approach than for the SAX approach, but the difference was not statistically significant. Due to limitations associated with the vascular conditions and operator experience, the LAX and OAX approaches are more difficult. In addition, because the operator cannot accurately judge the needle point and adjust the needle angle, the OAX approach is difficult.

Due to issues in the angle and direction of the needle, even if the puncture is successful, cannulation may fail, and when repeated adjustments are performed, the cannulation time and risk of adverse events 
increase. In the short-axis view, the artery appears to be a dark circle; when the needle exceeds the beam under ultrasound, only a part of the needle can be seen. In the process of cannulation, the needle easily penetrates the posterior wall and causes hematoma or ischemia[17]. The time of cannulation and the incidence of adverse events were higher than those with the LAX approach. Song et al.[7] also confirmed that the incidence of adverse events with the SAX approach was much higher than that with the LAX approach $(95.7 \%$ vs $18.0 \% ; P=0.000)$. Gopalasingam et al.[18] reported a high posterior wall puncture rate, indicating that the operators performed the SAX technique insufficiently.

The dynamic needle tip positioning technique (DNPT) has been confirmed to be a novel method to improve the success rate of ultrasound-guided radial artery cannulation in adults and children[19], but it is difficult for an anaesthesiologist who has no experience with ultrasound, and we did not choose to include this method in our comparison. The operators in our study had equivalent experience with the three approaches before commencing the study. The results might have been different if the participants had more specific experience with ultrasound-guided radial artery cannulation.

There are several limitations in this study.First, patients with relatively good vascular conditions were selected, and ultrasound guidance is of more value in difficult cases. The results may be different in complicated cases. Second,Clemmesen et al.[20] proposed that the DNPT method improved the success rate and reduced adverse events, and we did not compare the effects of these two approaches as this technology requires more capability for the operator.Third, each operator had only one chance to perform the operations, and there was no discussion of whether a second or multiple attempts affect the results. Fourth, every person's learning ability is different and might impacted the results, which needs to be confirmed by studies with larger sample sizes in the future.

In summary, compared with the LAX and OAX approaches, the SAX approach for ultrasound-guided cannulation of the radial artery yielded a higher success rate, shorter imaging time and shorter blood return time when it was performed by inexperienced anaesthesia residents. This approach was simple and easy to learn, yielded a high success rate, and is recommended for clinical teaching in the future.

\section{Abbreviations}

ASA: American Society of Anesthesiologists

$\mathrm{BMI}=$ Body mass index

SAX=short-axis/out-of-plane

LAX=long-axis/in-plane

OAX=oblique-axis/out-of-plane

$S D=$ standard deviation 
DNPT=dynamic needle tip positioning

\section{Declarations}

\section{Ethics approval and consent to participate}

This prospective study was approved by the Ethics Committee of Henan Provincial People's Hospital and written informed consent was obtained from patients.

\section{Consent for publication}

Not applicable

\section{Availability of data and materials}

The datasets used and analyzed during the current study are available from the corresponding author on reasonable request.

\section{Competing interests}

The authors declare that they have no competing interest.

\section{Funding}

None.

\section{Author's contribution}

YL C: manuscript and data analysis

HF: follow up

KK: data collection

JX S:data analysis

JQ Z: implementation of the study (IR)

MZ C: design and clinical studies

All authors read and approved the final version of the manuscript

Acknowledgments

Not applicable 


\section{References}

1. Ailon, J; Mourad, O; Chien, V; et al.Videos in clinical medicine. Ultrasound-guided insertion of a radial arterial catheter.[J].N Engl J Med.2014,371(15):e21

2. Gu, WJ; Wu, XD; Wang, F; et al.Ultrasound Guidance Facilitates Radial Artery Catheterization: A Metaanalysis With Trial Sequential Analysis of Randomized Controlled Trials.[J].Chest.2016,149(1):16679

3. Gao, YB; Yan, JH; Gao, FQ; et al.Effects of ultrasound-guided radial artery catheterization: an updated meta-analysis.[J].Am J Emerg Med.2015,33(1):50-5

4. Tang, L; Wang, F; Li, Y; et al.Ultrasound guidance for radial artery catheterization: an updated metaanalysis of randomized controlled trials.[J].PLoS One.2014,9(11):e111527

5. Bhattacharjee, S; Maitra, S; Baidya, DK; Comparison between ultrasound guided technique and digital palpation technique for radial artery cannulation in adult patients: An updated meta-analysis of randomized controlled trials.[J].J Clin Anesth.2018,47():54-59

6. Abdalla, UE; Elmaadawey, A; Kandeel, A; Oblique approach for ultrasound-guided radial artery catheterization vs transverse and longitudinal approaches, a randomized trial.[J].J Clin Anesth.2017,36():98-101

7. Song, IK; Choi, JY; Lee, JH; et al.Short-axis/out-of-plane or long-axis/in-plane ultrasound-guided arterial cannulation in children: A randomised controlled trial.[J].Eur $\mathrm{J}$ Anaesthesiol.:522-7

8. Melhuish, TM; White, LD; Optimal wrist positioning for radial arterial cannulation in adults: A systematic review and meta-analysis.[J].Am J Emerg Med.2016,34(12):2372-2378

9. Berk, D; Gurkan, Y; Kus, A; et al.Ultrasound-guided radial arterial cannulation: long axis/in-plane versus short axis/out-of-plane approaches?[J].J Clin Monit Comput.2013,27(3):319-24

10. Sethi, S; Maitra, S; Saini, V; et al.Comparison of short-axis out-of-plane versus long-axis in-plane ultrasound-guided radial arterial cannulation in adult patients: a randomized controlled trial.[J].J Anesth.2017,31(1):89-94

11. White, L; Vlok, R; Melhuish, T; Ultrasound-guided radial artery catheterization: A superior technique for expert clinicians?[J].Am Heart J.2019,2120:161-163

12. Ueda, K; Bayman, EO; Johnson, C; et al.A randomised controlled trial of radial artery cannulation guided by Doppler vs. palpation vs. ultrasound.[J].Anaesthesia.2015,70(9):1039-44

13. Blaivas, M; Brannam, L; Fernandez, E; Short-axis versus long-axis approaches for teaching ultrasound-guided vascular access on a new inanimate model.[J].Acad Emerg Med.2003,10(12):1307-11

14. Stone, MB; Moon, C; Sutijono, D; et al.Needle tip visualization during ultrasound-guided vascular access: short-axis vs long-axis approach.[J].Am J Emerg Med.2010,28(3):343-7

15. Phelan, M; Hagerty, D; The oblique view: an alternative approach for ultrasound-guided central line placement.[J].J Emerg Med.2009,37(4):403-8 
16. Sabouneh, R; Akiki, P; Al Bizri, A; et al.Ultrasound guided central line insertion in neonates: Pain score results from a prospective study.[J].J Neonatal Perinatal Med.2020,13(1):129-134

17. Stone, MB; Moon, C; Sutijono, D; et al.Needle tip visualization during ultrasound-guided vascular access: short-axis vs long-axis approach.[J].Am J Emerg Med.2010,28(3):343-7

18. Gopalasingam, N; Juhl-Olsen, P; Sloth, E; Posterior wall puncture during ultrasound-guided arterial cannulation suggests inadequate operator skills.[J].Eur J Anaesthesiol.2017,34(2):104

19. Liu, L; Tan, Y; Li, S; et al."Modified Dynamic Needle Tip Positioning" Short-Axis, Out-of-Plane, Ultrasound-Guided Radial Artery Cannulation in Neonates: A Randomized Controlled Trial.[J].Anesth Analg.2019,129(1):178-183

20. Clemmesen L, Knudsen L, Sloth E, et al.Dynamic needle tip positioning - ultrasound guidance for peripheral vascular access. A randomized, controlled and blinded study in phantoms performed by ultrasound novices.[J].Ultraschall Med.2012,33(7):E321-E325

\section{Figures}

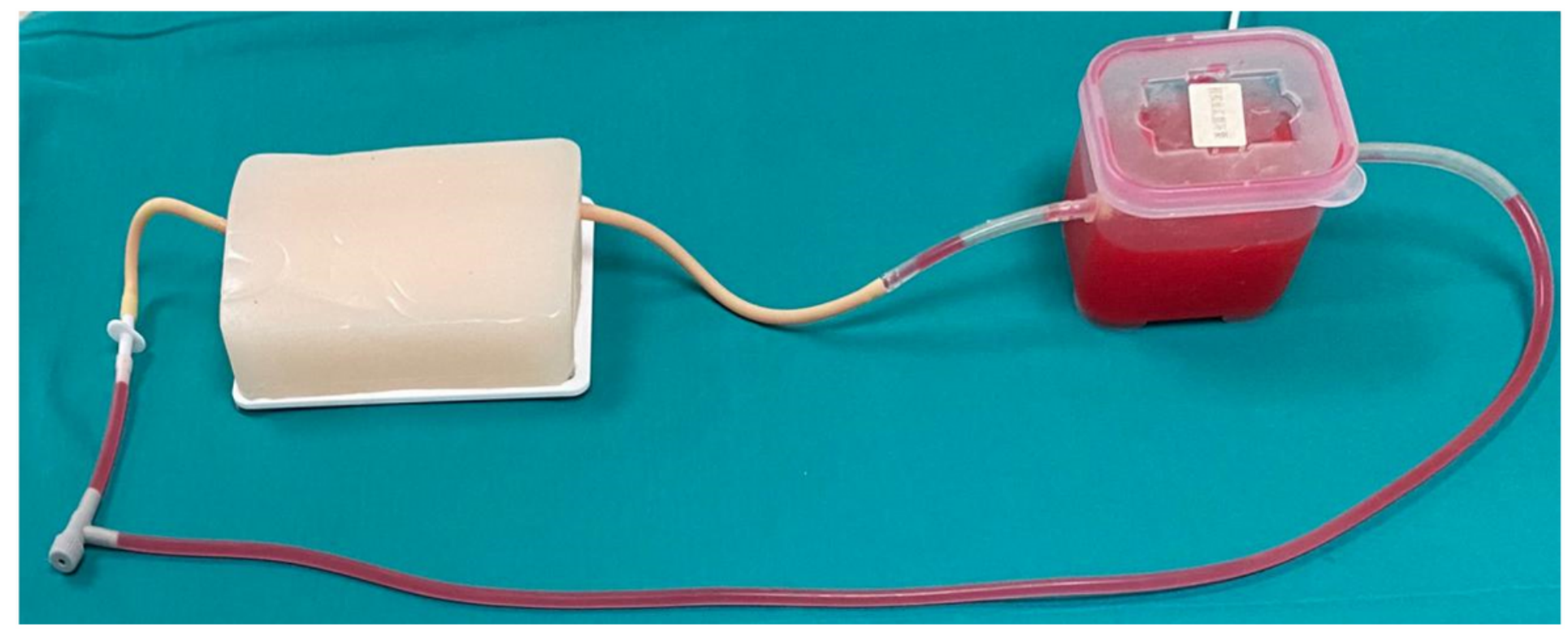

\section{Figure 1}

Radal artery model. 


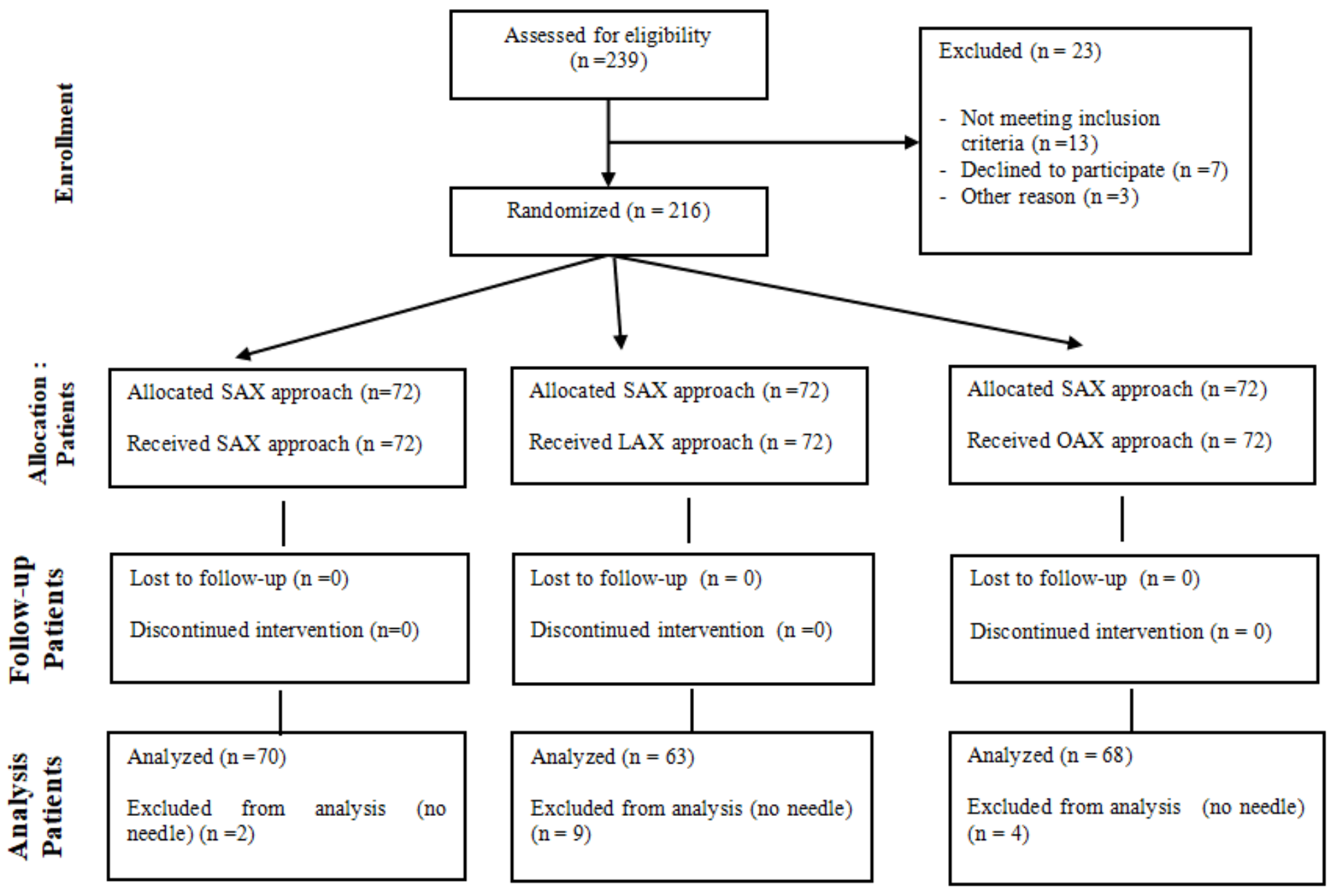

Figure 2

Study flow chart.

\section{Supplementary Files}

This is a list of supplementary files associated with this preprint. Click to download.

- CONSORTExtensionchecklist.docx

- CONSORTExtensionforAbstractsChecklist.doc 